\title{
Frequency of adverse reactions after subcutaneous allergen immunotherapy in children
}

\author{
Ayse Senay Sasihuseyinoglu', Dilek Doğruel', Derya Ufuk Altıntaș¹ \\ 1 Department of Pediatric Allergy and Immunology; Faculty of Medicine, Cukurova University, Adana, Türkiye \\ Ayse Senay Sasihuseyinoglu, ORCID: 0000-0003-4085-0256 \\ Dilek Doğruel, ORCID: 0000-0003-3972-7277 \\ Derya Ufuk Altıntaş, ORCID: 0000-0003-2090-5248
}

\begin{abstract}
Objective: Subcutaneous allergen immunotherapy (SCIT) is an effective treatment method for allergic rhinitis, asthma and venom allergy. The aim of this study was to evaluate the frequency of adverse reactions in children undergoing SCIT.
\end{abstract}

Methods: This retrospective study included patients that underwent SCIT in our clinic for a period of five years due to a diagnosis of Apis mellifera venom allergy or allergic asthma and/or rhinitis. 303 patients were divided into groups based on the form of SCIT administered and the presence of injection-related reactions.

Results: Mean age at the initiation of SCIT was 10 (range,
5-18) years old. SCIT for aeroallergens was administered to $289(95.4 \%)$ patients and SCIT for venom to $14(4.6 \%)$ patients. Local reactions were observed in 54 (17.8\%) and systemic reactions developed in $4(1.3 \%)$ patients. The local reactions mostly occurred after SCIT with Apis mellifera venom $(100 \%)$, followed by house dust mite $(20.6 \%)$, mold (16.7\%) and grass pollen (16.7\%).

Conclusion: Although SCIT is a safe treatment method used for allergic diseases, it must be administered only in centers with appropriate emergency equipment due to the risk of side effects.

Keywords: Subcutaneous immunotherapy, child, adverse reaction, allergy.
Correspondence: Ayse Senay Sasihuseyinoglu

Department of Pediatric Allergy and Immunology; Faculty of Medicine, Cukurova University, Adana, Turkey

E-mail: ssashuseyinoglu@yahoo.com.tr

Received: 16.10.2020; Accepted: 28.11.2020
Online available at: www.entupdatesjournal.org 


\section{Introduction}

Subcutaneous allergen immunotherapy (SCIT) is a treatment method based on the induction of clinical and immunological tolerance in clinically sensitized patients by the administration of allergen extract at an incremental dose beginning from a low dose and at doses causing no side effects. SCIT has been shown to be the sole treatment option affecting the natural course of allergic diseases. ${ }^{[1]}$ The common consensus on the utility of SCIT is that SCIT is a useful treatment method with proven effectiveness when used in well-selected patients and with appropriate indications and techniques. ${ }^{[2]}$ Moreover, SCIT could be a viable treatment option for patients that have been detected to have specific IgE via skin test and/or by in vitro methods and present with symptoms after exposure to natural allergen, in patients that present with significant side effects of drug therapy and in patients avoiding long-term drug use. ${ }^{[3]}$ Although SCIT is generally a safe therapy, it can cause undesirable side effects, from a simple local reaction to severe anaphylactic shock. ${ }^{[4]}$ The aim of the present study was to evaluate the frequency of adverse reactions in children undergoing SCIT due to aeroallergen and venom sensitivity in our clinic between 2007 and 2016.

\section{Materials and Methods}

\section{Study groups}

This retrospective study included patients that underwent SCIT with standard allergen extract in our clinic for a period of five years between January 2007 and December 2016 due to a diagnosis of Apis mellifera venom allergy or aller- gic asthma and/or rhinitis. Allergic asthma, allergic rhinitis and Apis mellifera venom allergy were diagnosed according to international guidelines. ${ }^{[5,6]}$ Patients were divided into groups based on the form of SCIT administered and the presence of injection-related reactions. Indications and contradictions for immunotherapy were evaluated based on the American Academy of Allergy, Asthma \& Immunology (AAAAI) guidelines. ${ }^{\left[{ }^{[3]}\right.}$ Written consent was obtained from each parent since the participants were between 5-18 years old prior to the initiation of immunotherapy. The study protocol was approved by the local institutional ethics committee (02.03.2018/75-44) and was performed in accordance with the Helsinki Declaration.

\section{Allergen immunotherapy}

Allergen immunotherapy was administered using standard allergen extracts available in Turkey, including Allergovit (Allergopharma, Reinbeck, Germany), ALK (ALK- Abellò, Madrid, Spain) or SAY (Stallargen, Antony Cedex, France). Table 1 presents the initial and maintenance doses of SCIT. The initial dose consisted of 1-4 injections per week for patients that underwent aeroallergen immunotherapy $(\mathrm{n}=289 ; 95.4 \%)$ and 1 vaccine per week for patients that underwent Apis mellifera venom immunotherapy ( $\mathrm{n}=14$; 4.6\%). The maintenance dose, which consisted of 100,000 standardized quality unit of vaccine (SQ-U) $1 \mathrm{ml}$, was commenced at months 4-6 and was administered monthly to all patients. No dose adjustment was required during pollen season and no grass pollen immunotherapy was administered to any patient. Prior to each injection, the patients were queried about their existing complaints and the com-

Table 1. Initial and maintenance doses of SCIT.

$\begin{array}{lcr} & \text { Initial dose } & \text { Maintenance dose } \\ \text { SAY (Stallargen) (Antony Cedex, France) } & 0.01 \mathrm{IR} 0.1 \mathrm{ml} & 10 \mathrm{IR} 0.8 \mathrm{ml} \\ \text { ALK (ALK-Abellò) (Madrid, Spain) } & 100 \mathrm{SQ}-\mathrm{U} 0.1 \mathrm{ml} & 100,000 \mathrm{SQ}-\mathrm{U} 1 \mathrm{ml} \\ \text { Allergopharma (Reinbeck, Germany) } & 5 \mathrm{TU} 0.2 \mathrm{ml} & 5,000 \mathrm{TU} 1 \mathrm{ml}\end{array}$

IR: Index of reactivity, ml: milliliter, SQ-U: standardized quality unit, TU: transforming unit 
plaints that emerged after the previous injection, and also a physical examination was performed for each patient. $\mathrm{Pa}-$ tients with asthma underwent a pulmonary function test prior to injection and for asthma patients that had existing complaints, injection was postponed by one week. All injections were administered subcutaneously by physicians and trained nurses under outpatient conditions.

\section{Classification of reactions}

Reactions associated with immunotherapy were classified as local and systemic based on their width and as early- and late-onset based on their time of onset. Local reaction was defined as the presence of swelling and redness at the injection site, and a large local reaction was defined as a local reaction greater than the size of the patient's palm at the injection site. ${ }^{[7]}$ Systemic reactions were classified based on the grading system proposed by the World Allergy Organization (WAO) ${ }^{[8]}$ Systemic reactions occurring within the first $30 \mathrm{~min}$ after injection were classified as early-onset and those occurring $30 \mathrm{~min}$ after injection were classified as late-onset systemic reactions. All patients were monitored for reactions for a minimum of $30 \mathrm{~min}$ after each injection. Patients that showed systemic or large local reactions were treated accordingly and their subsequent injection doses were re-adjusted.

\section{Statistical analysis}

Data were analyzed using SPSS for Windows Version 18.0 (SPSS Inc. Co., Chicago, IL, USA). Data were expressed as median, frequencies (\%) and percentages (\%).

\section{Results}

This study included 303 patients that underwent SCIT in our clinic for a period of five years between January 2007 and December 2016 and had complete clinical records. Mean follow-up period (which was calculated as the time from first presentation to our clinic to the initiation of SCIT) was 2.7 (range, 0.5-14) years. Mean age at the initiation of SCIT was 10 (range, 5-18) years old. Figure 1 presents the distribution of the forms of SCIT administered to our patients. SCIT was administered with a single allergen in $232(76.6 \%)$, two allergens in $66(21.8 \%)$ and three allergens in $5(1.7 \%)$ patients. Local reactions were observed in $54(17.8 \%)$ and systemic reactions developed in $4(1.3 \%)$ patients. Local reactions mostly occurred after SCIT with Apis mellifera venom (100\%), followed by

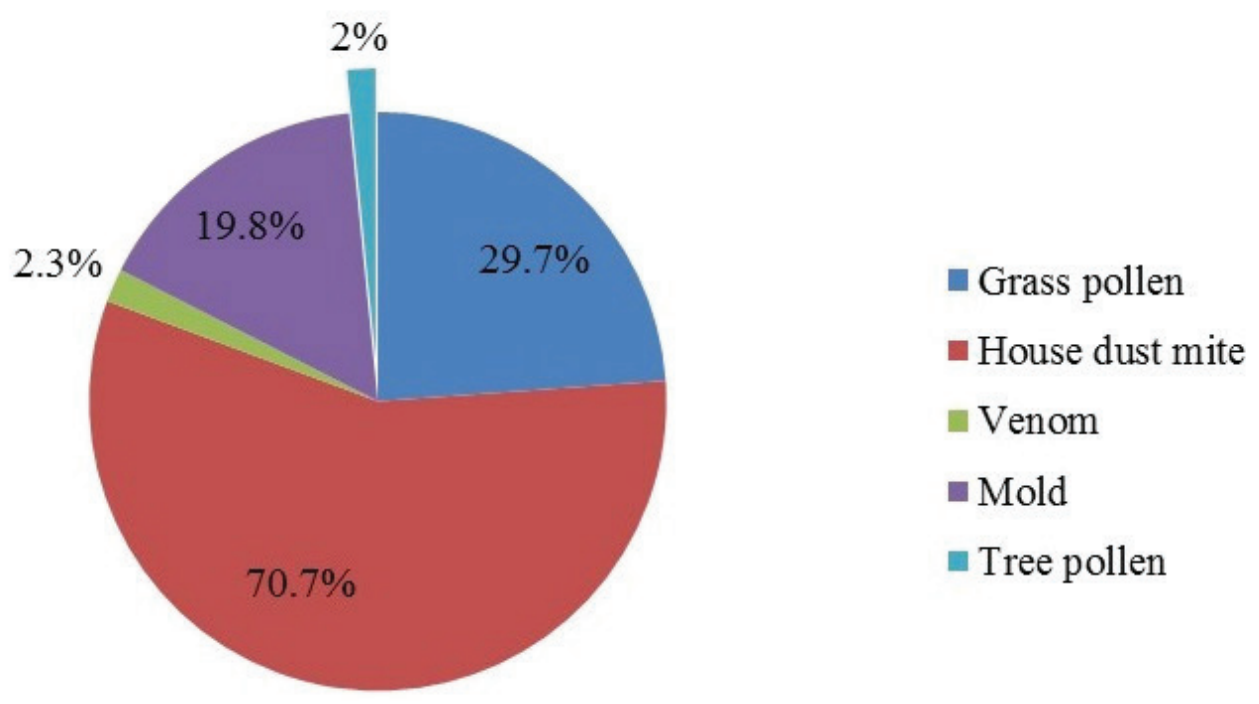

Figure 1. SCIT forms administered in the study. 
house dust mite (HDM) (20.6\%), mold (16.7\%) and grass pollen (16.7\%). Fifty-four local reactions were observed, including early-onset local reactions ( $\mathrm{n}=6 ; 11.1 \%)$, late-onset small reactions $(\mathrm{n}=15 ; 27.7 \%)$ and late-onset large reactions $(n=33 ; 61.1 \%)$. Of the local reactions, 20 (44.4\%) of them developed during the initial phase. Systemic reactions were generalized pruritis, angioedema, cough and wheezing. Systemic reactions were grade 1 and 2 , and none of them required adrenaline. Of the 4 systemic reactions, 1 reaction was observed during the initial phase and the remaining 3 reactions were observed in the maintenance phase. These reactions mostly occurred after SCIT with HDM (1.4\%), followed by grass pollen (1.1\%). Among these, two (50\%) patients had previously developed a local reaction.

\section{Discussion}

Allergen-specific immunotherapy essentially aims to overcome allergic inflammation by inducing T-cell tolerance to allergens similar to that of healthy individuals. ${ }^{\left[{ }^{[9}\right.}$ SCIT has been shown to be an effective treatment for allergic rhinitis and asthma and to prevent anaphylaxis to hymenoptera stings. ${ }^{[10]}$ The literature indicates that SCIT is a low-risk and well-tolerated treatment. ${ }^{[1]}$ In the literature, there is no consensus on lower and upper age limits for the initiation of immunotherapy. ${ }^{[12]}$ In addition, results of a recent study showed that the starting age for SCIT is not a risk factor for adverse effects in multivariate analysis. ${ }^{[13]}$ However, the administration of SCIT may result in local (erythema, swelling and itching at the injection site) and systemic reactions, of which local reactions can be seen in $26-82 \%$ and systemic reactions can be seen in $0.7-4 \%$ of patients. ${ }^{[14]}$ In our study, the prevalence of large local reactions was $10.89 \%$, which was consistent with the literature. In the literature, there are controversial findings regarding the ability of local reactions to predict local and systemic reactions following injections. La Shell et al. ${ }^{[15]}$ evaluated patients that underwent immunotherapy for fire ants and showed that the development of large local reactions was a risk factor for the development of systemic reactions. In our study, only two $(3.7 \%)$ patients with local reactions developed subsequent systemic reactions. Contrariwise, another study suggested that local reactions have no utility in predicting local reactions occurring after subsequent injections. ${ }^{[16]} \mathrm{A}$ previous Turkish study evaluated the prevalence of large local and systemic reactions in children undergoing SCIT and reported that the administration of injections during the initial or maintenance phase had no significant effect on the prevalence of large local reactions. ${ }^{[7]} \mathrm{It}$ is estimated that $1 / 160,000$ anaphylactic reactions occurred for every SCIT visit. ${ }^{[4]}$ SCIT-related systemic reactions in children have been reported to occur in $4 \%$ of all patients and in $0.1 \%$ of all injections. ${ }^{[17]} \mathrm{A}$ previous Turkish study evaluated 108 children that were followed up due to asthma and/or allergic rhinitis with pollen or HDM sensitivity and reported that the prevalence of early-onset systemic reactions after subcutaneous injection was $0.1 \%{ }^{[18]}$ Another study reported this rate as $0.3 \%$ in patients that received immunotherapy with pollen and venom. ${ }^{[7]}$ In our study, in line with the literature, the prevalence of systemic reactions was $1.3 \%$ and all of those reactions were observed within the first $30 \mathrm{~min}$ after injection. On the other hand, late-onset systemic reactions (anaphylaxis) have also been reported after SCIT. ${ }^{[19]}$ Similarly, other studies also reported that late-onset systemic reactions were observed in almost $50 \%$ of children undergoing SCIT and noted that those reactions were mostly mild and non-life-threatening. ${ }^{[20,21]}$ For these reasons, patients undergoing immunotherapy should be monitored for the possible risk of injection-related reactions for a minimum of $30 \mathrm{~min}$ after injections and should be trained accordingly. ${ }^{[3]}$

In conclusion, subcutaneous immunotherapy is a safe treatment for allergic patients. However, it should be administered by trained specialists under appropriate safety conditions due to the risk of injection-related reactions.

\section{Acknowledgments: None}

Ethics Committee Approval: The study protocol was approved by the local institutional ethics committee (Approval number: 02.03.2018/75-44). 
Informed Consent: A written consent was obtained from each parent since the participants were between 5-18 years old.

Author Contributions: Designing the study - DUA, ASS, DD; Collecting the data - ASS; Analysing the data ASS, DD; Writing the manuscript - ASS; Confirming the accuracy of the data and the analyses - DUA.

\section{References}

1. Tophof MA, Hermanns A, Adelt T, et al. Side effects during subcutaneous immunotherapy in children with allergic diseases. Pediatr Allergy Immunol 2018;29:267-74

2. Joint Task Force on Practice Parameters; American Academy of Allergy, Asthma and Immunology; American College of Allergy, Asthma and Immunology; Joint Council of Allergy, Asthma and Immunology. Allergen immunotherapy: a practice parameter second update. J Allergy Clin Immunol 2007;120:25-85

3. Cox L, Nelson H, Lockey R, et al. Allergen immunotherapy: a practice parameter third update. J Allergy Clin Immunol 2011;127:1-55.

4. Bernstein DI, Epstein TEG. Safety of allergen immunotherapy in North America from 2008-2017: Lessons learned from the ACAAI/AAAAI National Surveillance Study of adverse reactions to allergen immunotherapy. Allergy Asthma Proc 2020;41:108-11.

5. Brożek JL, Bousquet J, Agache I, et al. Allergic Rhinitis and its Impact on Asthma (ARIA) guidelines-2016 revision. J Allergy Clin Immunol 2017;140:950-8

6. Pedersen SE, Hurd SS, Lemanske RF Jr, et al. Global strategy for the diagnosis and management of asthma in children 5 years and younger. Pediatr Pulmonol 2011;46:1-17.

7. Azkur D, Vezir E, Civelek E, et al. The frequency of large local and systemic reactions after subcutaneous immunotherapy in children. [Article in Turkish] Asthma Allergy Immunol 2013;11:37-42.

8. Cox L, Larenas-Linnemann D, Lockey RF, Passalacqua G. Speaking the same language: The World Allergy Organization Subcutaneous Immunotherapy Systemic Reaction Grading System. J Allergy Clin Immunol 2010;125:569-74

9. Soyyiğit Ş, Sin BA. Asthma and Allergen Immunotherapy. [Article in Turkish] Güncel Göğüs Hastalıkları Serisi 2015;3:200-7.

10. Epstein TG, Liss GM, Murphy-Berendts K, Bernstein DI. Immediate and delayed-onset systemic reactions after subcutaneous immunotherapy injections: ACAAI/AAAAI surveillance study of subcutaneous immunotherapy: year 2. Ann Allergy Asthma Immunol 2011;107:426-31.
Conflict of Interest: The authors have no conflicts of interest to declare.

Financial Disclosure: The authors declare that this study has received no financial support.

11. Pfaar O, Bachert C, Bufe A, et al. Guideline on allergen-specific immunotherapy in IgE-mediated allergic diseases: S2k Guideline of the German Society for Allergology and Clinical Immunology (DGAKI), the Society for Pediatric Allergy and Environmental Medicine (GPA), the Medical Association of German Allergologists (AeDA), the Austrian Society for Allergy and Immunology (ÖGAI), the Swiss Society for Allergy and Immunology (SGAI), the German Society of Dermatology (DDG), the German Society of Oto- Rhino-Laryngology, Head and Neck Surgery (DGHNO-KHC), the German Society of Pediatrics and Adolescent Medicine (DGKJ), the Society for Pediatric Pneumology (GPP), the German Respiratory Society (DGP), the German Association of ENT Surgeons (BV-HNO), the Professional Federation of Paediatricians and Youth Doctors (BVKJ), the Federal Association of Pulmonologists (BDP) and the German Dermatologists Association (BVDD). Allergo J Int 2014;23:282-319.

12. Çekiç Ş, Sapan N. Allergen Specific Immunotherapy. [Article in Turkish] J Curr Pediatr 2015;13:46-55.

13. Gür Çetinkaya P, Esenboğa S, Uysal Soyer Ö, Tuncer A, Şekerel BE, Şahiner ÜM. Subcutaneous venom immunotherapy in children: Efficacy and safety. Ann Allergy Asthma Immunol 2018;120:424-8.

14. Adamic K, Zidarn M, Bajrovic N, Erzen R, Kopac P, Music E. The local and systemic side-effects of venom and inhaled-allergen subcutaneous immunotherapy. Wien Klin Wochenschr 2009;121:357-60.

15. La Shell MS, Calabria CW, Quinn JM. Imported fire ant field reaction and immunotherapy safety characteristics: the IFACS study. J Allergy Clin Immunol 2010;125:1294-9.

16. Calabria CW, Coop CA, Tankersley MS. The LOCAL Study: Local reactions do not predict local reactions in allergen immunotherapy. J Allergy Clin Immunol 2009;124:739-44.

17. Businco L, Zannino L, Cantani A, Corrias A, Fiocchi A, La Rosa M. Systemic reactions to specific immunotherapy in children with respiratory allergy: a prospective study. Pediatr Allergy Immunol 1995;6:44-7.

18. Can D, Demir E, Tanaç R, Gülen F, Yenigün A. Immediate adverse reactions to immunotherapy. J Investig Allergol Clin Immunol 2003;13:177-80, 
19. Şenbaba E, Çatal F, Topal E, Ermiştekin H, Yıldırım N, Sinanoğlu MS. Delayed severe systemic reaction (anaphylaxis) after subcutaneous immunotherapy injections: case report. [Article in Turkish] Asthma Allergy Immunol 2013;11:204-7.
20. Rank MA, Oslie CL, Krogman JL, Park MA, Li JT. Allergen immunotherapy safety: characterizing systemic reactions and identifying risk factors. Allergy Asthma Proc 2008;29:400-5.

21. Bernstein DI, Epstein T. Systemic reactions to subcutaneous allergen immunotherapy. Immunol Allergy Clin North Am 2011;31:241-9.

This is an open access article distributed under the terms of the Creative Commons Attribution-NonCommercial-NoDerivs 3.0 Unported (CC BY- NC-ND3.0) Licence (http://creativecommons.org/licenses/by-nc-nd/3.0/) which permits unrestricted noncommercial use, distribution, and reproduction in any medium, provided the original work is properly cited.

Please cite this article as: Sasihuseyinoglu A.S., Doğruel D., Altıntaş D.U. Frequency of adverse reactions after subcutaneous allergen immunotherapy in children. ENT Updates 2020;10(3): 396-401 